D.O.I.: $10.3895 / \mathrm{S} 1808-04482009000300010$

\title{
ANÁLISE COMPARATIVA DO BALANCED SCORECARD COM ALGUNS DOS PRINCIPAIS SISTEMAS DE MEDIÇÃO DE DESEMPENHO
}

\section{COMPARATIVE ANALYSIS OF THE BALANCED SCORECARD WITH SOME PERFORMANCE MEASUREMENT SYSTEMS}

\author{
Sidnei Vieira Marinho'; Paulo Mauricio Selig ${ }^{2}$ \\ ${ }^{1}$ Universidade do Vale do Itajaí - UNIVALI - Itajaí - Brasil \\ sidnei@univali.br \\ ${ }^{2}$ Universidade Federal de Santa Catarina - UFSC - Florianópolis - Brasil \\ selig@deps.ufsc.br
}

\begin{abstract}
Resumo
O objetivo deste artigo é apresentar um referencial teórico para obter um análise comparativa do Balanced Scorecard (BSC) com outros Sistemas de Medição de Desempenho (SMD `s). Este estudo torna-se importante devido ao avanço do campo da medição de desempenho que passa por uma verdadeira revolução. Muitos conceitos estão sendo revistos e os SMD `s tradicionais, baseados nos aspectos financeiros, não são apropriados a realidade organizacional das empresas. No entanto, o BSC como o SMD mais utilizado pelas organizações e aquele que apresenta o maior número de trabalhos publicados na área de medição de desempenho é efetivamente diferente dos demais SMD's?
\end{abstract}

Palavras-chave: estratégia; sistema de medição de desempenho; balanced scorecard.

\section{Estratégia}

Não há uma definição universal para o conceito de estratégia e é vasta a diversidade de termos utilizados, alguns autores utilizam metas e objetivos como parte da estratégia enquanto outros fazem claras distinções entre estratégias e objetivos. "Raramente a estratégia completa é escrita em qualquer lugar. Os processos usados para se chegar à estratégia total são tipicamente fragmentados, evolucionários e largamente intuitivos" (QUINN, 1992).

Mintzberg et al (2000) reconhecem cinco definições parciais que se inter-relacionam de forma a permitir às pessoas uma correta interpretação do conceito:

a) Estratégia é um plano: conjunto de diretrizes elaborado de forma consciente para atingir determinada meta no futuro; 
b) Estratégia é um padrão: consistência de comportamento ao longo do tempo, obtida pela análise de resultados do passado;

c) Estratégia é uma posição: ocupação de determinado nicho de mercado pelos produtos da organização;

d) Estratégia é uma perspectiva: é a forma fundamental de executar as atividades dentro da organização;

e) Estratégia é um truque: uma "manobra" específica para enganar a concorrência.

Estas definições podem competir entre si, pois há possibilidade de substituir umas às outras, mas normalmente os bons resultados surgem da utilização destes conceitos de forma conjunta, permitindo um entendimento amplo do conceito. As organizações desenvolvem planos para o futuro (estratégia pretendida) e extraem padrões do passado (estratégia realizada). Mintzberg et al (2000 afirmam que as estratégias pretendidas nem sempre são realizadas. As ações plenamente realizadas dentro do plano das estratégias pretendidas denominam-se estratégias deliberadas. O restante é denominado de estratégia não-realizada. Quando um padrão ou consistência ocorre e não era pretendido, surge a estratégia emergente, que fará parte da estratégia realizada. A figura 1 ilustra estas formas de concepção de estratégia.

Figura 1 - Formas de Concepção da Estratégia

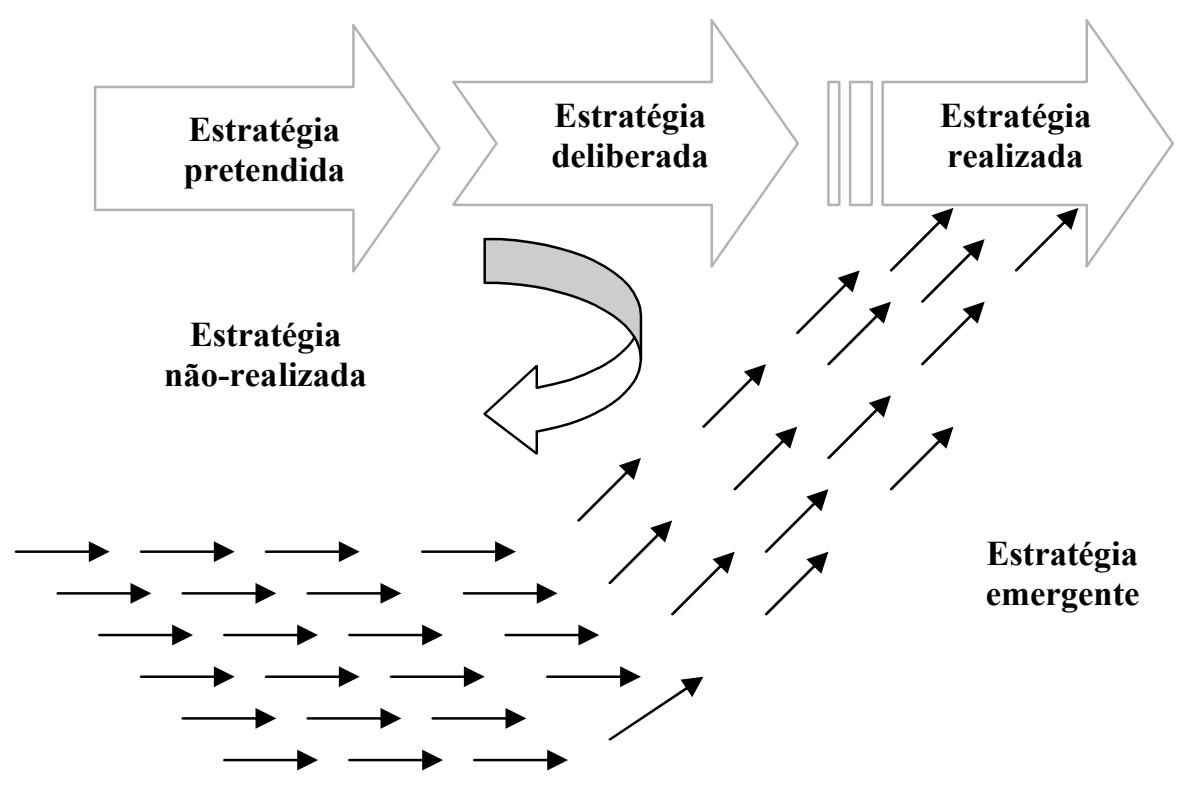

Fonte: Mintzberg (2004)

Pesquisas demonstram que os processos de formular estratégias têm seus limites (STACEY, 1993; CHAHARBAGHI; WILLIS, 1998; MINTZBERG et al, 2000). Portanto, nenhum plano pode ser completamente definido para um futuro incerto ou esperar pelas estratégias puramente emergentes, ausentes totalmente de intenções (BRUHN; LAGUNA, 2004). Mintzberg et al (2000) 
afirmam que "os estrategistas eficazes as misturam de maneira que reflitam as condições existentes, especialmente capacidade para prever e também a necessidade de reagir a eventos inesperados".

Segundo Mintzberg (2004, p. 230) "todo fracasso de implementação da estratégia é por definição um fracasso de formulação", pois a formulação e a implementação estratégicas interagem na prática, mesmo que não formalmente.

O processo de formulação da estratégia deve contemplar várias características das diferentes escolas, pois se torna difícil conceber uma estratégia negligenciando as imposições do ambiente; a força e o carisma do líder; a cultura organizacional; o espírito empreendedor; os modelos mentais; a inovação e a criatividade; a aprendizagem estratégica e etc.. O campo de estudo da estratégia é muito complexo e não permite a elaboração de um modelo ideal para a sua formulação, onde as melhores estratégias combinam deliberação e controle com flexibilidade e aprendizado.

Independente de como a estratégia da organização é formulada, ou seja, da escola de estratégia seguida pela organização, deve haver mecanismos de acompanhamento e avaliação da implantação das estratégias. Kaplan e Norton (2001) citam um estudo que revela que somente 10\% das estratégias formuladas com eficácia são implementadas com êxito e relatam que uma das causas é que as estratégias estão mudando e o mesmo não ocorre com as ferramentas para a mensuração das estratégias.

Kaplan e Norton (1997) identificaram 4 (quatro) barreiras específicas à implementação eficaz da estratégia:

a) Barreira no. 1: visão e estratégia não executáveis. Esta barreira ocorre quando a organização não consegue chegar a um consenso quanto à visão e estratégia pretendida. Com isso, os diversos grupos seguem suas próprias interpretações em relação à qualidade; melhoria contínua; reengenharia; empowerment, etc.. A ausência de integração não permite a consolidação de uma estratégia global.

b) Barreira $n^{0}$. 2: estratégia não associada às metas de departamentos, equipes e indivíduos. A ausência de desdobramento da estratégia para os departamentos, equipes e indivíduos faz com que o foco de desempenho fique limitado às ações táticas e de curto prazo, em detrimento da criação de capacidades que permitam a realização de metas estratégicas de longo prazo.

c) Barreira no. 3: estratégias não associadas à alocação de recursos. Muitas organizações não alinham os processos de planejamento estratégico e de orçamento. Com isso, os programas de ação não estão direcionados às prioridades estratégicas e as revisões periódicas analisam as divergências entre o real e o orçado e não com a evolução das metas estratégicas.

d) Barreira no. 4: feedback tático, não estratégico. A maioria dos sistemas gerenciais preocupa-se com a eficiência operacional do curto prazo, principalmente com medidas financeiras 
(real X planejado). A dedicação é mínima ao exame dos indicadores de implementação estratégica e assim, sem feedback não há como testar e aprender a estratégia na prática.

Alguns autores ressaltam a importância de um SMD para a implementação eficiente da estratégia em todos os níveis hierárquicos da organização (SINK; TUTTLE, 1993; HRONEC, 1994; MOREIRA, 1996; KAPLAN; NORTON, 1997, 2001, 2004; PORTER, 1992; NANDHAKUMAR, 1996; DELONG; ROCKART, 1992; BARROW, 1990; BURKAN, 1991; VOLONINO; WATSON, 1992; WATSON, 1992; DRUCKER, 2000; SIMONS; DÁVILA, 2000; GEROLAMO, 2003; FERNANDES, 2002 e 2003; BITITCI et al, 1997; MARTINS, 1998; MUSCAT; FLEURY, 1993).

A economia atual baseada em ativos intangíveis exige Sistemas de Medição de Desempenho (SMD`s) que descrevam os ativos com base no conhecimento e as estratégias criadoras de valor. $\mathrm{Na}$ ausência destes SMD`s, as organizações têm dificuldade em gerenciar o que não consegue descrever ou mensurar.

Neste contexto, o próximo item contextualiza os Sistemas de Medição de Desempenho (SMD`s) como forma de facilitar o processo de implementação da estratégia.

\section{Sistemas de Medição de Desempenho}

Entre o século XIV e o século XIX surgiram as primeiras práticas de contabilidade custos e de sistemas de produção que permitiam as organizações controlar e planejar a atuação no mercado da época.

Os primeiros relatos sobre Sistemas de Medição de Desempenho (SMD`s), no século XX, ocorreram na França com a adoção do Tableaux de Bord como forma das organizações entenderem as relações entre ações e resultados, este sistema teve sua utilização ampliada com a crise de 1929.

Peter Drucker lançou em 1954 a Administração por Objetivos (APO) que por meio de uma série de passos permitia a organização conferir os resultados das metas estabelecidas.

Na década de 70 vários sistemas de medição surgiram com o objetivo de vincular estratégias de mercado e retorno financeiro. Nos anos 80 destaque para a obra de Michael Porter sobre planejamento estratégico e como as organizações deveriam posicionar-se no mercado e desvincular estratégia de eficiência operacional.

Analisando os artigos publicados nas décadas de 80 e 90, observa-se um aumento significativo nas pesquisas sobre SMD`s, após a publicação do primeiro artigo sobre BSC por Kaplan e Norton em 1992. Outro ponto que merece destaque é o acréscimo de artigos e teses publicados com o objetivo de quantificar o número de SMD's existentes na literatura.

Kaplan e Norton em 1992 incorporaram o conceito de sistema de gestão estratégica por meio da obra Balanced Scorecard. Apesar da dificuldade de acesso a todas as obras relacionadas a 
Sistemas de Medição de Desempenho, percebe-se a evolução decorrente das necessidades de mercado impostas pelas evoluções do mundo dos negócios (KAPLAN; NORTON, 1997).

Figueiredo et al. (2005) com base nos resultados da revisão da literatura definem o conceito de Sistema de Medição de Desempenho (SMD) como sendo:

O conjunto de pessoas, processos, métodos, ferramentas e indicadores estruturados para coletar, descrever e representar dados com a finalidade de gerar informações sobre múltiplas dimensões de desempenho, para seus usuários dos diferentes níveis hierárquicos. Com base nas informações geradas, os usuários podem avaliar o desempenho de equipes, atividades, processos e da própria organização, para tomar decisões e executar ações para a melhoria do desempenho (inspirado em CLARK, 1995; BITITCI et al, 2000; NEELY et al, 2002).

Pandolfi (2005) ressalta que "sistemas de gestão devem basear-se em sistemas de indicadores que mostrem coerência entre os indicadores mensurados, fatores críticos, estratégias competitivas e objetivos estratégicos adotados" (BITITCI et al, 1997; MARTINS, 1998; KAPLAN; NORTON, 1997; MUSCAT; FLEURY, 1993).

Para que um SMD seja efetivamente implementado pela organização, os funcionários devem estar motivados para as mudanças decorrentes da análise dos resultados e para a possibilidade de um aprendizado completamente novo que obrigue a esquecer os antigos modelos mentais. Além disso, verifica-se que a avaliação de desempenho normalmente é vista como uma forma de controle e punição dos funcionários, onde as ações corretivas estão associadas à rigidez e falta de sensibilidade dos responsáveis pelo comando.

O sucesso de um SMD parece fazer parte de um processo desgastante e demorado. Sendo assim, as organizações devem estar cientes que funcionários felizes; infra-estrutura adequada; treinamento para as novas habilidades estratégicas; recompensas pelas sugestões implementadas; tempo para assimilar as mudanças; etc. fazem parte do diferencial das empresas com visão no futuro.

Neely et al (1995) e Martins (1998) apresentam as características que um sistema de mensuração de desempenho deve possuir:

a) Ser congruente com a estratégia competitiva;

b) Ter medidas financeiras e não financeiras;

c) Direcionar e suportar a melhoria contínua;

d) Identificar tendências e progressos;

e) Facilitar o entendimento das relações de causa e efeito;

f) Ser inteligível para os funcionários;

g) Abranger todo o processo, desde o fornecedor até o cliente;

h) Ter as informações disponíveis em tempo real, para toda a organização; 


\section{i) Ser dinâmico;}

j) Influenciar a atitude dos funcionários;

k) Avaliar o grupo e não o indivíduo.

Martins e Salerno (1998) acreditam que outras pesquisas precisam ser desenvolvidas com o intuito de sistematizar as informações geradas pelos SMD`s, pois não há abrangência e dinamismo suficiente para proporcionar a integração vertical e horizontal das organizações.

Sink e Tuttle (1993) citam alguns paradigmas da medição de desempenho:

a) a precisão é essencial à medição útil. A finalidade básica da medição de desempenho é gerar para a organização parâmetro de comparação, sem necessidade de exatidão nos resultados, pois as variações não impedem o processo de melhoria;

b) as medidas assustam as pessoas. O sistema de medição não pode ser utilizado como forma de intimidação dos funcionários para obter melhores resultados. Os funcionários gostam de obter feedback sobre o trabalho realizado e a organização deve aproveitar este fator como forma de compartilhar uma visão sobre os objetivos do sistema de medição;

c) focalizar o desempenho em único indicador. A complexidade organizacional não permite que o desempenho esteja expresso em um único indicador. A organização deve buscar um conjunto de indicadores que permita analisar e melhorar os fatores críticos de sucesso;

d) foco exagerado na produtividade da mão de obra. Apesar da redução da ênfase na mão de obra no processo de tomada de decisão da produção de determinado produto, este item continua tendo destaque nas organizações;

e) medidas subjetivas não são confiáveis. A medida de desempenho associada a percepções e atitudes está bem desenvolvida e pode levar a medidas confiáveis e válidas, especialmente na prestação de serviços e em organizações de trabalho intelectual;

f) os padrões funcionam como teto para a desempenho. Não existem níveis absolutos de desempenho. Sendo assim, as organizações com foco no futuro e no processo de melhoria contínua precisam mudar este paradigma.

Para garantir uma eficiente implementação do Sistema de Medição de Desempenho, as organizações precisam atingir algumas metas (BERLINER; BRIMSON, 1992; HRONEC, 1994):

a) Desenvolver um sistema hierárquico de medições que ligue o negócio, a planta e o desempenho do chão-de-fábrica;

b) Voltar às metas da organização e selecionar os tipos de medidas de desempenho que suportam as metas;

c) Identificação dos processos da empresa; 
d) Compreensão dos clientes (internos e externos) e seus desejos;

e) Determinar o que medir, por quê medir, quando medir e como medir;

f) Validar as medidas (pelas pessoas de dentro do processo) - submeter às medições de desempenho a todos os interessados afetados;

g) Começar a medição e emitir os relatórios correspondentes - captar, monitorar, analisar e divulgar as medidas de desempenho;

h) Avaliar a efetividade das medidas implementadas;

i) Analisar e melhorar continuamente as medições de desempenho.

Miranda (2005) utiliza os modelos de Chang e Young (1999) e Santos et al (2002) para definir as etapas de um SMD com ênfase na melhoria contínua dos processos da organização, visando relacionar os esforços das atividades do dia-a-dia com a visão e a missão organizacional. As etapas do método são: planejamento, medição, análise e melhoria (vide figura 2).

No planejamento, torna-se necessário o levantamento e a compreensão de todos os processos da organização, pois permite a correta identificação das áreas-chave da organização, e os respectivos indicadores e metas.

A definição dos atributos de medição de desempenho compõe a segunda etapa do modelo, que corresponde aos seguintes passos: determinar a coleta de dados; o rastreamento dos dados e maneiras de executar o feedback.

$\mathrm{Na}$ análise ocorre a definição de um plano de ação, com objetivos e táticas, visando alcançar as metas. Por último, a etapa de melhoria, provendo informações para reavaliar e reestruturar o SMD e divulgando os resultados na organização. 
Figura 2 - Método de Desenvolvimento de um SMD
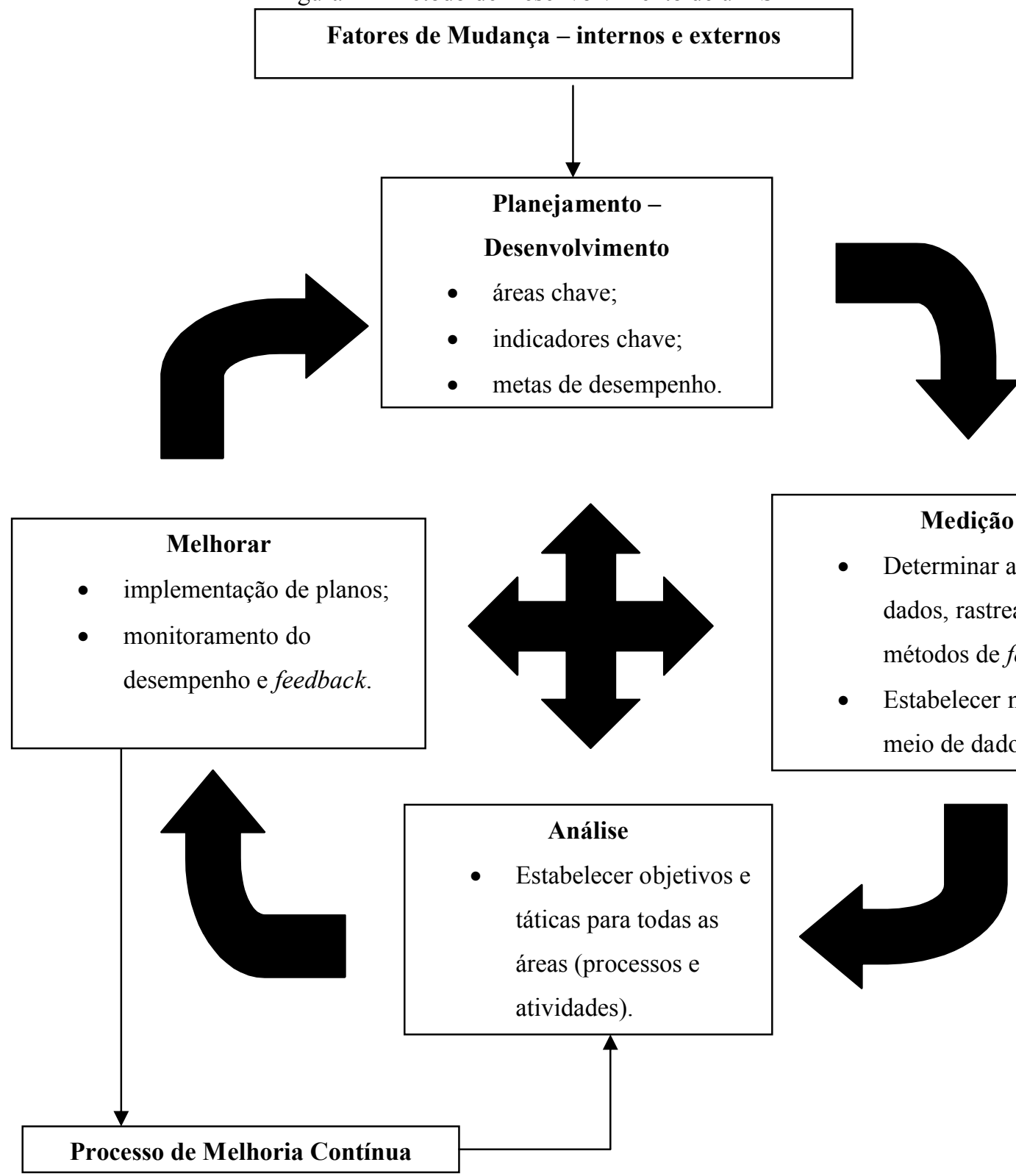

Fonte: Miranda (2005)

No quadro 1 são apresentados alguns sistemas de medição de desempenho encontrados na literatura específica da área. Estudos completos podem ser encontrados em Martins (1998), Figueiredo (2003) e Petri (2005).

Quadro 1 - Exemplos de Sistemas de Medição de Desempenho

\begin{tabular}{|c|c|c|}
\hline SISTEMA/ABORDAGEM & AUTOR & ANO \\
\hline Tableaux de Bord de Gestion & Satet e Voraz (apud Malo) & 1932 \\
\hline Administração por Objetivos & Drucker (apud Lodi) & 1954 \\
\hline Gerenciamento pelas Diretrizes & Akao & 1960 \\
\hline Melhoria de Performance & Sink e Tuttle & 1989 \\
\hline Pirâmide de Performance (SMART) & Mcnair, Lynch e Cross & 1990 \\
\hline $\begin{array}{c}\text { Brignall, Fritzgerald, Johnston, Silvestro e } \\
\text { Voss }\end{array}$ & Brignall, Fritzgerald, Johnston, Silvestro e Voss & 1991 \\
\hline
\end{tabular}




\begin{tabular}{|c|c|c|}
\hline Juran & Juran & 1992 \\
\hline Campos & Campos & 1992 \\
\hline Três Níveis do Desempenho & Rummler e Brache & 1992 \\
\hline Balanced Scorecard & Kaplan e Norton & 1992 \\
\hline $\begin{array}{c}\text { PEMP (Progresso Efetivo e Medição de } \\
\text { Performance) }\end{array}$ & Adams e Roberts & 1993 \\
\hline Gerenciamento de Processos Empresariais & Harrington & 1993 \\
\hline Ernst e Young & Ostrenga & 1994 \\
\hline Quantum & Hronec & 1994 \\
\hline Human Capital Intelligence & Fitz-Enz & 1994 \\
\hline Navegador Skandia & Edvinsson e Malone & 1995 \\
\hline Moreira & Moreira & 1996 \\
\hline Métricas de Desempenho & Universidade da Califórnia - USA-DoE & 1996 \\
\hline Navegador do Capital Intelectual & Stewart & 1996 \\
\hline Macroprocesso de uma Organização & Brown & 1996 \\
\hline Family Nevada Quality Fórum & Family Nevada Quality Fórum - USA-DoD & 1997 \\
\hline Sistêmica & Sandia National Laboratories - USA-DoE & 1997 \\
\hline $\begin{array}{c}\text { Gerenciamento Total da Melhoria } \\
\text { Contínua }\end{array}$ & Harrington & 1997 \\
\hline Monitor de Ativos Intangíveis & Sveiby & 1997 \\
\hline Value Chain Scoreboard & Lev & 2001 \\
\hline Performance Prism & Neely, Adams e Kennerly & 2002 \\
\hline
\end{tabular}

Fonte: autoria própria (2006)

Vale ressaltar, que no quadro 1 são apresentadas as datas de publicação dos primeiros artigos ou datas consideradas pelos autores como sendo o marco de consolidação do sistema/abordagem. A data mencionada no Tableaux de Bord de Gestion é referente ao ano de popularização do termo que ficou conhecido na literatura, já que este SMD começou a ser desenvolvido no final do século XIX.

\section{Balanced Scorecard}

Em meados de 1990, o Instituto Nolan Norton, uma unidade de pesquisa da KPMG, patrocinou um estudo, de duração de um ano, intitulado "Measuring Performance in the Organization of the Future”. A motivação para realizar o referido estudo foi acreditar que os métodos tradicionalmente utilizados para a mensuração do desempenho empresarial estavam se tornando obsoletos por utilizarem apenas indicadores contábeis e financeiros. Com o desenvolvimento das pesquisas, chegou-se a uma proposta que foi denominada de Balanced Scorecard (BSC). Este SMD procura traduzir a visão e a estratégia da organização em objetivos, 
medidas (ou indicadores), metas e iniciativas sob a ótica de quatro perspectivas distintas: financeira, dos clientes, dos processos internos, e do aprendizado e crescimento (KAPLAN; NORTON, 1997; MÂSIH et al, 2001).

O BSC é um sistema de gestão que desdobra a visão e a estratégia em objetivos e indicadores em quatro perspectivas conforme a Figura 3.

Figura 3 - Balanced Scorecard

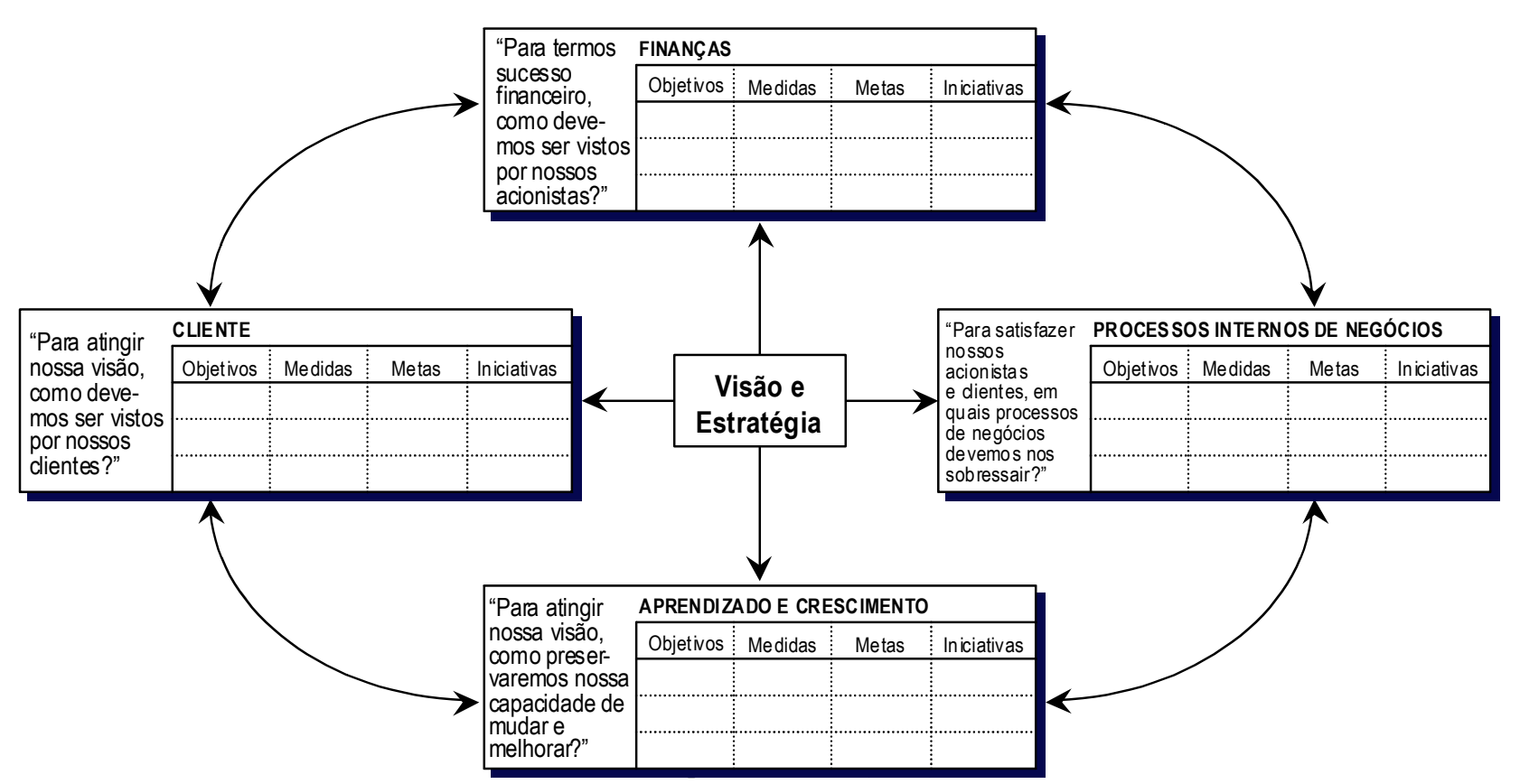

Fonte: Kaplan e Norton (2001)

O BSC define a estratégia como sendo um conjunto de hipóteses de causa e efeito, onde a implementação da estratégia é a compreensão das hipóteses por todos na organização, o alinhamento dos recursos com as hipóteses, o teste contínuo das hipóteses e sua adaptação em tempo real, conforme as necessidades (KAPLAN; NORTON, 2001).

As quatro perspectivas propostas por Kaplan e Norton (2001) estão presentes na grande maioria dos estudos de caso, mas dependendo da análise do ambiente e da estratégia adotada podese agregar mais perspectivas. Soares Júnior e Prochnik (2005) analisam o caso de uma unidade de negócio da Siemens Brasil, que atua no setor de telecomunicações, que acrescentou a perspectiva regulatória, em função da forte influência da ANATEL. Outro caso na Siemens estava relacionado à mudança constante da tecnologia e, por essa razão, acrescentou, ao seu BSC, a perspectiva de tecnologia e inovação.

A experiência adquirida em mais de dez anos de aplicação do BSC permitiu a Kaplan e Norton acompanhar a evolução do ponto de vista conceitual. Em 1992, os autores defendiam o uso de amplo conjunto de indicadores distribuídos pelas quatro perspectivas. Na seqüência, observou-se 
que os indicadores deveriam monitorar a estratégia, pois antes de medir era necessário perguntar aos executivos: Quais são os objetivos estratégicos da organização? A definição dos indicadores passou para um papel secundário, pois quando havia consenso sobre os objetivos almejados, a organização poderia mudar os indicadores insatisfatórios sem a necessidade de reavaliar a estratégia adotada. Ou seja; os objetivos continuam os mesmos, ainda que os indicadores evoluíssem pela experiência ou nova fonte de dados. O foco nos objetivos permitiu que a estratégia fosse descrita mediante a identificação das hipóteses de causa e efeito entre os objetivos nas quatro perspectivas. Esse diagrama é chamado de Mapa Estratégico (KAPLAN; NORTON, 2004).

“O mapa estratégico revelou-se inovação tão importante quanto o próprio Balanced Scorecard original" (KAPLAN; NORTON, 2004, p.9).

Vale destacar, os diferentes sentidos que Kaplan e Norton mencionam sobre o conceito:

Quadro 2 - As Diferentes Funções do Mapa Estratégico

\begin{tabular}{l} 
1. Mostrar o destino estratégico: "O mapa estratégico possibilita que uma organização descreva e ilustre, \\
em linguagem clara e geral, seus objetivos, iniciativas e alvos; os indicadores utilizados para avaliar seu \\
despenho e as conexões, que são o fundamento da direção estratégica." \\
\hline 2. Destacar o valor do capital intelectual: "O mapa estratégico, ajustado à estratégia específica da \\
organização, descreve como os ativos intangíveis impulsionam melhorias de desempenho dos processo \\
internos da organização, que exercem o máximo de alavancagem no fornecimento de valor para os clientes, \\
os acionistas e as comunidades." \\
\hline 3. Representar visualmente a estratégia; "O mapa estratégico é a representação visual da estratégia, \\
mostrando numa única página como os objetivos nas quatro perspectivas se integram e combinam para \\
descrever a estratégia." \\
\hline \begin{tabular}{l} 
4. Ligar o trabalho individual à estratégia: "Com o mapa estratégico, os empregados têm uma \\
representação visual de como suas funções se conectam com os objetivos gerais da empresa, ao mesmo \\
tempo em que os gerentes compreendem com mais clareza a estratégia e identificam os meios para detectar e \\
corrigir os desvios de rumo." \\
\hline 5. Demonstrar o fluxo de valor: "Os objetivos nas quatro perspectivas são conectados uns aos outros por \\
relações de causa e efeito. A partir do topo, parte-se da hipótese de que os resultados financeiros só serão \\
alcançados se os clientes-alvo estiverem satisfeitos." \\
\hline 6. Reforçar a importância do conhecimento: "O fundamento de qualquer mapa estratégico é a perspectiva \\
de aprendizado e crescimento, que define as competências e habilidades essenciais, as tecnologias e a cultura \\
organizacional necessárias para suportar a estratégia da empresa."
\end{tabular} \\
\hline
\end{tabular}

Fonte: Kaplan e Norton apud Herrero Filho (2005)

A prática do BSC pelas organizações e a necessidade do desdobramento da estratégia na definição de objetivos, indicadores, metas e iniciativas mostrou que esta etapa superou as expectativas do roteiro de implementação da estratégia. Sendo assim, este processo tornou-se um valioso instrumento para assegurar a aprendizagem estratégica por todos na organização.

Schwarz, Amiden e Pinho (2005) destacam que o processo de desdobramento da estratégia permite que a organização alcance resultados em termos de: alinhamento dentro da organização, criação de sinergias, criação de valor para a organização, comunicação da estratégia, integração entre corporação; áreas de negócios e unidades de apoio, uniformização de indicadores ao longo da organização, acompanhamento do desempenho local, efetiva implementação da estratégia e uniformização de esforços. 
A FPNQ (2001) identifica três métodos de correlação que podem auxiliar na formatação das relações de causa e efeito entre os objetivos estratégicos do BSC:

a) correlação visual: a qualificação técnica da equipe para analisar relatórios, tabelas e indicadores permite identificar visualmente os graus de influência entre as variáveis analisadas e garantir em determinadas situações o bom resultado da análise;

b) correlação estatística: a aplicação de softwares estatísticos permite identificar quantitativamente os graus de influência entre as variáveis analisadas; embora não elimine a interpretação dos dados como no método anterior;

c) simulação dinâmica: softwares especializados (Excel, Powersim, iThink, Vensim, etc.) permitem utilizar combinações hipotéticas para prever os possíveis desdobramentos dos cenários futuros, sendo que a comparação com os dados reais gera a identificação de padrões de comportamento.

Sendo assim, os mapas estratégicos não são a única alternativa para desdobramento de estratégia, algumas organizações utilizam outras ferramentas conjunta ou isoladamente para auxiliar neste processo: mapas de sucesso; discussão dos "fatores críticos de sucesso" para alcance dos objetivos estratégicos; identificação de contribuições das unidades do nível inferior para com os objetivos da unidade do nível superior; mapas de aprendizado (learning maps); árvores estratégicas de causa e efeito; modelo causal; desdobramento do modelo causal; "pais e filhos"; negative branch; diagrama de construção de indicadores de desempenho estratégicos; diagrama para a construção de relacionamentos entre as medidas de desempenho; mapa cognitivo e analytic hierarchy process (NEELY et al, 2002; CUSTODIO, 2004; SCHWARZ et al, 2005; KAPLAN; NORTON, 2001 e 2004).

A utilização de métodos analíticos para a experimentação das hipóteses dos mapas estratégicos, permite a adaptação da estratégia, conforme a necessidade e a dinâmica dos stakeholders envolvidos.

Kaplan e Norton (2001) citam algumas empresas que reforçam a validade das hipóteses por meio de simulação e análises estatísticas. Uma divisão da Brown e Root Energy Services utiliza análise de correlações para examinar as relações entre os indicadores, por exemplo; correlação entre o moral dos funcionários e nível de satisfação dos clientes. O Grupo Bal desenvolveu um modelo dinâmico de simulação, para analisar os loops de feedbacks e atrasos. Becker et al (2001) destacam a experiência da Sears, uma das primeiras empresas a quantificar as hipóteses do mapa estratégico, utilizando a análise estatística para verificar o impacto das ações na avaliação dos indicadores.

A concepção dos mapas estratégicos está fundamentada na Dinâmica de Sistemas (System Dynamics), onde as relações de causa e efeito permitem a discussão de diferentes visões com 
relação à interdependência dos elementos chaves de um processo (FERNANDES, 2002). “Tem como pressuposto o fato de que o comportamento de um sistema é determinado por sua estrutura, que é composta por loops de feedbak e delays" (MEDEIROS Jr.; SOUZA NETO, 2004, p. 4).

Entretanto, apesar do avanço da utilização dos mapas estratégicos na lógica sistêmica, verifica-se que o BSC comunica os objetivos estratégicos de forma linear e estática, pois desconsidera os efeitos circulares dos feedbacks e delays (tempo de espera) da estratégia. O desdobramento unidirecional do BSC não mantém conexão com as restrições impostas pelo sistema, como disponibilidade de capital financeiro ou estrutura organizacional (FERNANDES, 2002; LINARD et al, 2002).

A causalidade unidirecional dos mapas estratégicos do BSC fica evidente quando se considera que todas as perspectivas estão orientadas para a perspectiva financeira. Não existe possibilidade de influência da dimensão financeira sobre as outras. Além disso, os mapas não captam as variações necessárias para se ter uma visão dinâmica de um sistema, possibilitando apenas uma "fotografia" da estratégia (RICHMOND apud MEDEIROS Jr.; SOUZA NETO, 2004).

\section{Metodologia}

Este artigo é parte dos resultados de uma pesquisa de 5 anos que culminou na realização de uma tese de doutorado. A pesquisa envolveu o levantamento bibliográfico elaborado a partir de livros, artigos de periódicos, material disponibilizado na Internet e a análise de exemplos que estimulou a compreensão do problema em questão, ou seja; existe efetivamente diferença entre o Balanced Scorecard e outros Sistemas de Medição de Desempenho?

O próximo tópico apresentará uma Análise Comparativa do Balanced Scorecard com alguns dos principais Sistemas de Medição de Desempenho.

\section{Análise Comparativa do Balanced Scorecard com alguns dos principais Sistemas de Medição de Desempenho}

O elevado número de Sistemas de Medição de Desempenho e a impossibilidade de detalhar os conceitos e características envolvidos nos mesmos, faz com que haja a necessidade de estabelecer critérios para a escolha dos SMD's para realização da análise comprativa. Sendo assim, o presente trabalho irá utilizar os seguintes SMD's: 
a) O Tableaux de Bord de Gestion (1932); Administração por Objetivos (1954) e o Gerenciamento pelas Diretrizes (1960) pela importância histórica no estudo da gestão baseada na avaliação de desempenho;

b) O Navegador Skandia que representa os sistemas de medição de capital intelectual, que apresenta elevada quantidade de publicações e tornou-se referência importante na consolidação da teoria da Gestão do Conhecimento;

c) O Performance Prism pela representatividade acadêmica comprovada pelo número de citações em artigos, este SMD fica atrás apenas do Balanced Scorecard de Kaplan e Norton (MARR; SCHIUMA, 2003).

Alguns autores não percebem diferenças entre o Tableaux de Bord e o Balanced Scorecard (STRENGER, 1997; EPSTEIN; MANZONI, 1997; MENDONZA; ZRIHEN; 1999). No entanto, Costa (2001) afirma que as diferenças entre as aplicações e os estilos de gestão mencionados pelos referidos autores refletem diferenças de concepções e análises:

Os diferentes estilos de gestão constituem a aplicação prática de diferentes modelos teóricos. Diferenças de aplicação refletem acima de tudo, diferenças na compreensão a respeito do instrumento que está sendo utilizado. Modelos com diferentes ênfases são diferentes modelos. Balanced Scorecard e Tableaux de Bord, portanto, são, em termos teóricos, diferentes modelos que fundamentam diferentes práticas/estilos. (COSTA, 2001, p. 89).

Para Costa (2001) o Balanced Scorecard é uma contribuição original em relação ao Tableaux de Bord, porque:

a) Repensa temas antigos, mostrando a vinculação entre pontos vitais que não estavam claramente vinculados;

b) Mostra como estes conhecimentos estabelecidos podem ser articulados para serem aplicados aos tempos modernos, para atender às novas exigências;

c) Aumentando, assim, a compreensão do tema "vinculação entre estratégia e operação" e aperfeiçoando a literatura científica sobre o tema.

Os setores-chave da Administração por Objetivos são semelhantes aos temas estratégicos das perspectivas do BSC. Dinesh e Palmer (1998) ressaltam esta similaridade ao afirmarem que ambos são baseados em objetivos estratégicos e formulados por meio de um processo interativo que envolve os vários níveis da organização. Além disso, destacam que as medidas do Balanced Scorecard vinculadas a incentivos e recompensas tornam-se uma ferramenta útil (Kaplan e Norton, 1997). Por outro lado, pesquisas mostram o mesmo da Administração por Objetivos (DEWEY, 1995; SHAW; SCHNEIER, 1995). 
Uma diferença perceptível entre os dois modelos é o grau de clareza. O APO é um sistema aberto baseado na colaboração de todos dentro da organização para determinação de objetivos e medidas. O BSC também utiliza está lógica, no entanto é mais detalhado que o APO, pois descreve seus objetivos em quatro perspectivas (finanças, clientes, processos internos e aprendizado e crescimento). Este detalhamento permite aos usuários do BSC especial atenção para os objetivos não-financeiros.

A análise do Balanced Scorecard e do Gerenciamento pelas Diretrizes mostra que existem pontos de complementaridade entre estes sistemas de gestão. Na implementação da estratégia, a função do BSC é a tradução e o acompanhamento da estratégia no longo prazo e o Gerenciamento das Diretrizes é responsável em transformar a estratégia em tarefa de todos, focando nos objetivos, metas e iniciativas do curto prazo. Sendo assim, o aprendizado estratégico seria resultado das hipóteses estratégicas (BSC) e da discussão do desempenho de cada meta (GPD) (REDI, 2003). No entanto, existem dúvidas de como operacionalizar dois sistemas de gestão, buscando o melhor de cada um, para traduzir os objetivos estratégicos em metas operacionais (WITCHER, 2003).

Pesquisas recentes relacionam o Navegador Skandia com o Balanced Scorecard, segue uma mostra de alguns relatos que ajudam a entender os pontos comuns e conflitantes dos dois SMD's.

Macdonald (2003) enfatiza que a divulgação de indicadores do Navegador Skandia ocorre por meio de relatório que é distribuído para os funcionários, enquanto o BSC desdobra os indicadores para todos os níveis da organização, principalmente no nível tático e operacional.

Nessa linha, Cordeiro (2002) ressalta que o BSC parece utilizar uma abordagem mais racional na determinação dos indicadores, fazendo com que a metodologia tenha um número menor de indicadores em relação à quantidade utilizada no Navegador Skandia.

Bukh et al (2002) comparam a aplicação do Navegador Skandia com o BSC em uma organização voltada para produção de softwares na Dinamarca. O estudo de caso permitiu concluir que o modelo de CI tem foco nas competências da organização enquanto o BSC reforça a utilização de indicadores de controle gerencial. Apesar disso, os autores acreditam que estes SMD's podem ser utilizados de forma integrada. Wu (2005); Andriessen (2004); Walker e MacDonald (2001); Hagood e Friedman (2002) realizaram outros estudos e reforçam esta tese.

Para Olve et al (2001), o Navegador Skandia utiliza a mesma lógica do Balanced Scorecard com o acréscimo de um quinto foco, o dos recursos humanos, harmonizando assim o BSC com a categoria de capital intelectual. Ao justificar o uso desta perspectiva, a Skandia enfatiza que os recursos humanos devem estar associados aos processos de negócios de uma organização, pois se reforçam mutuamente. Sendo que, o alto desempenho da organização é o resultado do escore alto em ambas perspectivas. 
Para Neely et al (2002) o Performance Prism complementa o BSC ao analisar os usuários finais, empregados, fornecedores, reguladores, os grupos de pressão ou comunidades locais, ou seja; reforçam a tese que estes stakeholders podem ter um grande impacto na organização e na condução de suas atividades. Os autores ressaltam ainda que o BSC da forma como foi concebido está orientado para a criação de valor para os acionistas.

\title{
6. Conclusões
}

O BSC não apresenta nenhum conceito novo que permita considerá-lo inédito dentro dos estudos na área de medição de desempenho. No entanto, o BSC repensa alguns temas que estavam sendo abordados equivocadamente por outros SMD`s. O artigo mostrou que o BSC evoluiu dentro de sua própria concepção original e que não possui grandes divergências teóricas com outros SMD`s, apenas consegue obter um melhor resultado na compreensão do desdobramento da estratégia em ações operacionais.

A relação de causa e efeito entre os objetivos nas quatro perspectivas, representado pelo mapa estratégico, transformou-se em um eficaz mecanismo de compreensão estratégica. Além disso, ressalta-se a significativa melhora da variável motivacional que permite aos funcionários a efetiva participação no processo de desdobramento da estratégia.

Neste contexto, pode-se afirmar que o BSC consegue, apesar da ausência de originalidade teórica, ser mais eficiente que os outros SMD`s.

\begin{abstract}
This article introduces a theoretical framework for a comparative analysis of the Balanced Scorecard (BSC) with others Performance Measurement Systems. This study is important to the advancement of the field of performance measurement that goes through a revolution. Many concepts are being reviewed and the Performance Measurement Systems traditional, based on the financial aspects, are not appropriate organizational reality of companies. However, the BSC as the Measurement System used by most organizations and one that has the highest number of published papers in the area of performance measurement is effectively different from the others Performance Measurement Systems?
\end{abstract}

Key-words: strategy; performance measurement systems; balanced scorecard.

\section{Referências}

ANDRIESSEN, D. Intellectual capital valuation and measurement: classifying the state-of-the-art. Journal of Intellectual Capital. Vol. 5, No. 2. pp. 230-242. 2004.

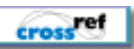

BARROW, C. Implementing an executive information system: sevem steps for success. International Journal of Information Systems Management. v. 7, n. 2, pp. 41-52. 1990. 
BECKER, Brian E. HUSELID, Mark A. e ULRICH, Dave. Gestão estratégica de pessoas com "scorecard": interligando pessoas, estratégia e performance. Rio de Janeiro: Campus. 2001.

BITITCI, U. S. CARRIE, A. S. McDEVITT, L. Integrated performance measurement systems: a development guide. International Journal of Operations \& Production Management. v. 17, n. 5, pp. 692-704. 1997.

cross ${ }^{\text {ref }}$

BITITCI, U. S. TURNER, T. BEGEMANN, C. Dynamics of performance measurement systems. International Journal of Operations \& Production Management. v. 20, n. 6, pp. 692-704. 2000.

cross $r$ ef

BRUHN, Paulo Roberto Lima. e LAGUNA, Gilmar Lemes._Processo Estratégico Integrado - Proposta de um modelo de abordagem estratégica baseado no Balanced Scorecard. In: Anais do XVII Congresso Latino-Americano de Estratégias. Itapema - SC. 2004.

BUKH, P. N. JOHANSEN, M. R. MOURITSEN, J. Multiple integrated performance management systems: IC and BSC in a software company. Singapore Management Review. 24.3. pp. 21-33. 2002.

BURKAN, W. C. Executive informations systems: from proposal to implementation. New York: Van Nostrand Reinhold. 1991.

CHAHARBAGHI, K. WILLIS, R. Strategy: the missing link between continuous revolution and constant evolution. International Journal of Operations \& Production Management. v.18, n.9/10, pp.1017-1027. 1998.

cross ${ }^{\text {ref }}$

CHANG, R. Y. YOUNG, P. D. Measuring organizational improvement impact. Califórnia: Jossey-Bass-Pfeiffer. 1999.

CLARK, L. A. Development, application, and evaluation of an organizational performance measurement system. Tese de Doutorado. Virginia Polytech Institute and State University. Blacksburg. 1995.

CORDEIRO, José Vicente Bandeira de Mello. Reflexões sobre a avaliação do desempenho empresarial na era da informação: uma comparação entre a gestão do capital intelectual e o Balanced Scorecard. Revista da FAE. v.5, n.2. pp.61-76.maio/ago. 2002.

COSTA, Ana Paula Paulino da. Contabilidade gerencial: um estudo sobre a contribuição do balanced scorecard. Dissertação de Mestrado. Universidade de São Paulo (USP). 2001.

CUSTODIO, Flavio Augusto. Uso do data mining no estabelecimento de relacionamentos entre medidas de desempenho. Dissertação de Mestrado. Universidade Federal de São Carlos (UFSCar). 2004.

DELONG, D. W. ROCKART J. F. Identifying the attributes of successful executive support system implementation. Chichester: John Wiley e Sons. 1992.

DEWEY, B. Aligning work and rewards. Management Review. v. 84, n. 2, pp. 19-23. 1995. 
DINESH, David. PALMER, Elaine. Management by objectives and the Balanced Scorecard: will Rome fall again?. Management Decision. 36/6, pp. 363-369. 1998.

cross ref

DRUCKER, Peter F. As informações de que os executivos realmente precisam. In Medindo o desempenho empresarial. Rio de Janeiro: Campus. 2000.

EPSTEIN, Marc J. MANZONI, Jean-Francois. The balanced scorecard and tableau de bord: translating strategy into action. Management Accounting; 79, 2; pp. 28. agosto.1997.

FERNANDES, Amarildo da Cruz . Mapas Estratégicos do Balanced Scorecard: Contribuições Ao Seu Desenvolvimento. In Anais do XXII Encontro Nacional de Engenharia de Produção. Curitiba. 2002.

FERNANDES, Amarildo da Cruz. Scorecard Dinâmico: em direção a integração da dinâmica de sistemas com o Balanced Scorecard. Tese de Doutorado. Universidade Federal do Rio de Janeiro (UFRJ). 2003.

FIGUEIREDO, Moacyr Amaral Domingues. Sistemas de medição de desempenho organizacional: um modelo para auxiliar a sua auto-avaliação. Tese de Doutorado. Universidade Federal do Rio de Janeiro (UFRJ). 2003.

FIGUEIREDO, Moacyr Amaral Domingues. MACEDO-SOARES, Teresia Diana Lewe van Aduard de. FUKS, Saul. FIGUEIREDO, Lívia Cavalcanti. Definição de atributos desejáveis para auxiliar a auto-avaliação dos novos sistemas de medição de desempenho organizacional. Gestão \& Produção. v.12, n.2, pp.305-315, mai.-ago. 2005.

Fundação do Prêmio Nacional de Qualidade (FNPQ). Planejamento do sistema de medição do desempenho global: relatório do comitê temático. 2001.

GEROLAMO, Mateus Cecílio. Proposta de sistematização para o processo de gestão de melhorias e mudanças de desempenho. Dissertação de Mestrado. Universidade de São Paulo (USP). 2003.

HAGOOD, W. O. e FRIEDMAN, L. Using the balanced scorecard to measure the performance of your HR information system. Public Personnel Management. Vol. 31, No. 4. pp. 543-557. 2002.

HERRERO FILHO, Emílio. Balanced scorecard e a gestão estratégica: uma abordagem prática. Rio de Janeiro: Campus. 2005.

HRONEC, S. M. Sinais vitais: usando medidas de desempenho da qualidade, tempo e custos para traçar a rota para o futuro de sua empresa. São Paulo: Makron Books. 1994.

KAPLAN, Robert. S. e NORTON, David. P. Balanced scorecard: A estratégia em ação. Rio de Janeiro: Campus. 1997.

KAPLAN, Robert. S. e NORTON, David. P. Organização orientada para a estratégia: como as empresas que adotam o balanced scorecard prosperam no novo ambiente de negócios. Rio de Janeiro: Campus. 2001.

KAPLAN, Robert. S. e NORTON, David. P. Mapas estratégicos - balanced scorecard: convertendo ativos intangíveis em resultados tangíveis. Rio de Janeiro: Campus. 2004. 
LINARD, Keith. FLEMING, Cherylne. DVORSKY, Lubomir. System dynamics as the link between corporate vision and key performance indicators. 20th System Dynamics International Conference. Italy. 2002.

MACDONALD, Raquel Purnhagen. Um sistema de avaliação de desempenho fundamentado no balanced scorecard: implantação em uma empresa alemã de médio porte. Dissertação de Mestrado. Universidade Federal de Santa Catarina (UFSC). 2003.

MARR, Bernard. SCHIUMA. Gianni. Business performance measurement - past, present and future. Management Decision. 41/8, pp. 680-687. 2003.

cross ${ }^{\text {ref }}$

MARTINS, R. A. SALERNO, M. S. Sistema de medição de desempenho: uma revisão da literatura. Boletim Técnico da Escola Politécnica. BT/PRO/063. Universidade de São Paulo. 1998.

MARTINS, R. A. Sistemas de medição de desempenho: um modelo para estruturação do uso. 1998. Tese de Doutorado. Universidade de São Paulo (USP). São Paulo. 1998.

MÂSIH, Rogério Teixeira. MARINHO, Sidnei Vieira. SELIG, Paulo Maurício. A utilização do balanced scorecard em empresas sem fins lucrativos: um estudo de caso. In Anais do XXI Encontro Nacional de Engenharia de Produção. Salvador. 2001.

MEDEIROS JÚNIOR, Josué Vitor de. SOUSA NETO, Manoel Veras de. Aplicação do método scorecard dinâmico na gestão estratégica de pequenas empresas. In: Anais do XVII Congresso Latino-Americano de Estratégias. Itapema SC. 2004.

MENDONZA, Carla. ZHIREN, Robert. Le tableau de bord: en V.O. ou en version américaine? - comparaison entre le tableau de bord et le balanced scorecard. Revue Française de Comptabilité. Número 309. pp. 60-66. Março de 1999.

MINTZBERG, Henry. AHLSTRAND, Bruce. e LAMPEL, Joseph. Safári de estratégia: um roteiro pela selva do planejamento estratégico. Porto Alegre: Bookman. 2000.

MINTZBERG, Henry. Ascensão e queda do planejamento estratégico. Porto Alegre: Bookman. 2004.

MIRANDA, Raíssa Alvares de Matos. Fatores que afetam o suporte fornecido pela medição de desempenho ao processo de melhoria contínua em empresas certificadas ISO 9001. Dissertação de Mestrado. Universidade Federal de São Carlos (UFSCar). 2005.

MOREIRA, Daniel Augusto. Dimensões do Desempenho em Manufatura e Serviço. São Paulo: Pioneira. 1996.

MUSCAT, A. R. N. FLEURY, A. C. C. Indicadores de qualidade e produtividade na indústria brasileira. Revista Indicadores de Qualidade e Produtividade. v. 1, n. 2, pp. 81-107. 1993.

NANDHAKUMAR, J. Design for success: critical success factors in executive information systems development. International Journal of Information Systems. Operations Research Society. pp. 62-72. 1996. 
NEELY, A. D. GREGORY, M. J. PLATTS, K. W. Performance measurement system design - a literature review and research agenda. International Journal of Operations \& Production Management. v. 15, n. 4, pp. 80-116. 1995.

cross ref

NEELY, Andy. ADAMS, Chris. KENNERLY, Mike. The performance prism: the scorecard for measuring and managing business success. Prentice Hall. 2002.

OLVE, Nils-Göran. ROY, Jan. WETTER, Magnus. Condutores de performance: um guia prático para o uso do balanced scorecard. Rio de Janeiro: Qualitymark. 2001.

PANDOLFI, Marcos. Sistemas de medição e avaliação de desempenho organizacional: contribuição para a gestão de metas globais a partir de performances individuais. Tese de Doutorado. Universidade de São Paulo (USP). 2005.

PETRI, Sérgio Murilo. Modelo para apoiar a avaliação das abordagens de gestão de desempenho e sugerir aperfeiçoamentos: sob a ótica construtivista. Tese de Doutorado. Universidade Federal de Santa Catarina (UFSC). 2005.

PORTER, Michael E. Vantagem competitiva: criando e sustentando um desempenho superior. Rio de Janeiro: Campus, 1992.

QUINN, James Brian. Strategic change: "logical incrementalism". In: Mintzberg, Henry e Quinn, James Brian.[Org.] The strategy process - concepts and contexts. Englewood Cliffs: Prentice Hall, pp. 96-104. 1992.

REDI, Renata. Modelo de implementação da estratégia através do uso integrado do balanced scorecard e do gerenciamento pelas diretrizes. Dissertação de Mestrado. Universidade Federal de Santa Catarina (UFSC). 2003.

SANTOS, S. P. BELTON, V. HOWICK, S. Adding value to performance measurement by using system dynamics and multicriteria analysis. International Journal of Operations e Production Management. v. 22, nº 11, pp.1216-1272. 2002.

cross ref

SCHWARZ, Fanny. AMIDEN, Maria Auxiliadora Moraes. e PINHO, Luís Cláudio. Alinhamento e desdobramento da estratégia. In COUTINHO, André Ribeiro. KALLÁS, David. (organizadores) Gestão estratégica: experiências e lições de empresas brasileiras. Rio de Janeiro: Campus. 2005.

SHAW, D. SCHNEIER, C. Team measurement and rewards: how some companies are getting it right. Human Resource Planning. v.18, n. 3, pp. 34-49. 1995.

SIMONS, Robert. DÁVILA, Antonio. Qual a magnitude do retorno sobre a gestão?. In Medindo o desempenho empresarial. Rio de Janeiro: Campus. 2000.

SINK, D. S. e TUTTLE, T. C. Planejamento e medição para a performance. Rio de Janeiro: Qualitymark. 1993.

SOARES JÚNIOR, Heles; PROCCHNIK, Victor. Experiências comparadas de implantação do Balanced Scorecard no Brasil. Disponível em: http://www.ie.ufrj.br/cadeiasprodutivas/pdfs/implantacao_do_balanced_scorecard_no_brasil.pdf . Acessado em 06/12/2007. 
STACEY, R. Strategy as Order Emerging from Chaos. Long Range Planning, v.26, n.1, pp.10-17. 1993.

cross ref

STRENGER. Ellen Weisman. Dashboards, Smashboards. Trustee. Chicago. Vol. 50. Iss. 4. pp. 9. Abril 1997.

VOLONINO, L. A. e WATSON, H. J. The strategic business objectives method for guiding executive information systems development. In: WATSON, H. Kelly e HOUDESHEL, G. Executive information systems: emergence, development e impact. New York: John Wiley. pp.145-159. 1992.

WALKER, G. e MACDONALD, R. Designing and implementing an HR scorecard. Human Resource Management. Vol. 20, No. 4. pp.365-377. 2001.

cross ref

WATSON, H. J. Avoiding hidden EIS pitfalls - a case study: what you see isn`t always what you get. In: WATSON, H. Kelly e HOUDESHEL, G. Executive information systems: emergence, development e impact. New York: John Wiley. pp.237-244. 1992.

WITCHER, Barry J. Policy management of strategy (hoshin kanri). Strategic Change. 12, 2. Mar/Apr 2003.

WU, Anne. The integration between balanced scorecard and intellectual capital. Journal of Intellectual Capital. 6, 2. pp. 267. 2005.

\section{Dados dos autores:}

Nome completo: Sidnei Vieira Marinho

Filiação institucional: Universidade do Vale do Itajaí (UNIVALI)

Departamento: Programa de Mestrado em Administração (PMA)

Função ou cargo ocupado: Professor

Endereço completo para correspondência (bairro, cidade, estado, país e CEP):

Rodovia Amaro Antônio Vieira, 2797

Apto 404 Bloco B; Itacorubi; Florianópolis - SC; CEP: 88034-101

Telefones para contato: (48) 99800630

e-mail:sidnei@univali.br

Nome completo: Paulo Mauricio Selig

Filiação institucional: Universidade Federal de Santa Catarina (UFSC)

Departamento: Departamento de Engenharia de Produção e Sistemas (DEPS) 
Função ou cargo ocupado: Professor

Endereço completo para correspondência (bairro, cidade, estado, país e CEP):

Rodovia Amaro Antônio Vieira, 2797

Apto 404 Bloco B; Itacorubi; Florianópolis - SC; CEP: 88034-101

Telefones para contato: (48) 99720561

e-mail:selig@deps.ufsc.br

Recebido para publicação em: 09/06/2009

Aceito para publicação em: 08/09/2009 